\title{
Idiopathic pulmonary fibrosis: a holistic approach to disease management in the antifibrotic age
}

\author{
Jonathon Shaw, Tracey Marshall, Helen Morris, Conal Hayton, Nazia Chaudhuri \\ North West Interstitial Lung Disease Unit, Manchester University Foundation Trust, Wythenshawe, Manchester, UK \\ Contributions: (I) Conception and design: J Shaw, T Marshall, H Morris, N Chaudhuri; (II) Administrative support: C Hayton; (III) Provision of study \\ materials or patients: N Chaudhuri, T Marshall, H Morris; (IV) Collection and assembly of data: All authors; (V) Data analysis and interpretation: \\ All authors; (VI) Manuscript writing: All authors; (VII) Final approval of manuscript: All authors. \\ Correspondence to: Conal Hayton. North West Interstitial Lung Disease Unit, Manchester University Foundation Trust, Southmoor Road, \\ Wythenshawe, Manchester M23 9LT, UK. Email: conalhayton@doctors.org.uk.
}

\begin{abstract}
Idiopathic pulmonary fibrosis (IPF) is the most common cause of interstitial lung disease (ILD) and carries a worse prognosis than many cancers. Until recently, there were no active treatment options available for patients with IPF, meaning palliation or lung transplantation in selected patients were the only options. The management of IPF has changed dramatically over the last decade with the advent of two antifibrotic agents; pirfenidone and nintedanib. These new agents have been shown to reduce decline in lung function and pirfenidone has been shown to reduce mortality. The changing landscape of IPF diagnosis and management present a number of issues that may be encountered including management of side effects related to antifibrotic therapy. This article aims to give an overview of the holistic approach to the management of patients with IPF, including antifibrotic management, symptom management and the invaluable role of the ILD specialist nurse.
\end{abstract}

Keywords: Idiopathic pulmonary fibrosis (IPF); interstitial lung diseases (ILDs); therapeutics; dyspnea; cough

Submitted Oct 05, 2017. Accepted for publication Oct 17, 2017.

doi: $10.21037 /$ jtd.2017.10.111

View this article at: http://dx.doi.org/ 10.21037/jtd.2017.10.111

\section{Introduction}

Idiopathic pulmonary fibrosis (IPF) is the most common cause of interstitial lung disease (ILD) and is characterized by progressive fibrosis of the lungs. It typically presents in the sixth or seventh decade causing cough, fatigue and breathlessness (1). A recent British Lung Foundation (BLF) report suggests that the incidence is higher than published reports at 6,000 new cases per year and that 32,500 people in the United Kingdom (UK) live with IPF (2). The global incidence of IPF is between 2.8-9.3 per 100,000 (3) and prevalence is increasing by $5 \%$ per year (4). The prognosis of IPF is poor and is worse than most cancers with a median survival from diagnosis of 2.5-3.5 years (5). In 2012 over 5,000 people died of IPF in the UK accounting for $1 \%$ of all deaths and $4.6 \%$ of respiratory deaths in the UK (2).

Until recently, there were no active treatment options available for patients with IPF, meaning palliation or lung transplantation in selected patients were the only options. However, with the introduction of anti-fibrotic agents including pirfenidone and most recently nintedanib there has been a dramatic field change in the management of IPF. These represent the first potentially disease modifying agents in this devastating disease while also bringing new challenges with management of side effect profiles. This article aims to provide an overview of management of IPF with reference to antifibrotic choice, management of side effects, symptom control and the integral role of the ILD specialist nurse in coordinating care.

\section{Disease-modifying management}

\section{Historical treatment}

Prior to the advent of antifibrotic therapies, immunosuppressive 
regimens were the mainstay of IPF treatment. Triple therapy with prednisolone, azathioprine and $\mathrm{N}$-acetylcysteine (NAC) became the treatment of choice following the publication of the IFIGENIA study that suggested this combination slowed the decline in lung function (6). However, a subsequent study, the PANTHER study published in 2012 caused a dramatic shift in the management of IPF (7). PANTHER showed that traditional triple therapy resulted in excess mortality when compared to placebo. This led to a period of no credible treatment options other than palliation or lung transplantation in highly selective cases. However, the introduction of evidence-based antifibrotic therapies has again given hope for patients with this devastating disease.

\section{Antifibrotic therapy: pirfenidone}

The progressive nature of IPF can be clinically assessed by decline in lung function over time. This is depicted as decline in forced vital capacity (FVC), diffusion capacity of the lungs for carbon monoxide (DLCO) and total lung capacity (TLC). FVC decline is the only reliable surrogate marker for mortality in IPF and is thus the primary end-point in clinical trials of treatments in IPF (8).

Pirfenidone is an immunosuppressive agent designed to specifically target the underlying disease mechanism in IPF. Although its mechanism of action is not fully understood, it is likely that pirfenidone prevents fibroblast proliferation and therefore prevents the release of pro-fibrotic and proinflammatory mediators (9).

Two randomised control trials (RCT) underpin the evidence base for pirfenidone in IPF. The CAPACITY-1 and CAPACITY-2 (10) trials compared decline in FVC in patients taking pirfenidone or placebo in mild to moderate IPF (FVC >50\%). Pooled analysis of these studies demonstrated that pirfenidone significantly reduced decline of FVC at 72 weeks ( $8.5 \%$ vs. $11 \%$ for placebo, $\mathrm{P}=0.005$ ). The study also demonstrated that significantly fewer patients taking pirfenidone had a FVC decline $>10 \%$, a marker of poor prognosis in IPF. The second ASCEND study (11) was requested by the United States Food and Drug Administration (US FDA) due to the inconsistent findings in the CAPACITY trials in meeting the primary endpoint. This RCT corroborated the initial findings, again showing a reduction in FVC decline in patients using pirfenidone compared to placebo. In a pre-specified pooled analysis of CAPACITY and ASCEND, both all-cause and IPF related mortality were significantly reduced with the use of pirfenidone (hazard ratio 0.52 and 0.32 respectively) (12).

Pirfenidone received approval from the US FDA in 2014, having previously received approval from the European Medicines Agency (EMA) in 2010. The National Institute of Health and Care Excellence (NICE) approved use of pirfenidone in the UK in April 2013 (13). Prescribing is restricted by NICE to ILD specialist centres in England and is licensed for patients with an FVC between 50-80\% predicted. NICE mandates that pirfenidone treatment should be stopped if there is a decline in FVC >10\% within a 12-month period whilst on treatment. This technology appraisal is a complex cost-effective decision rather than on clinical effectiveness. There is now evidence in posthoc analysis of these trials that pirfenidone significantly reduced the proportion of patients having a $\geq 10 \%$ decline in FVC or death in patients with an FVC above $80 \%$ (14). The EMA recommend that pirfenidone is used in "mild to moderate IPF" but do not attach any FVC restriction to this. There are no limitations attached to the FDA approval of pirfenidone.

\section{Antifibrotic therapy: nintedanib}

Nintedanib is a tyrosine kinase inhibitor and causes inhibition of intracellular growth factor signalling. This pathway is thought to be involved in the development of fibrosis and its inhibition leads to a reduction in profibrotic mediators (15). Nintedanib is also licensed for the treatment of advanced non-small cell lung cancer. The evidence for nintedanib in IPF primarily comes from two double-blinded placebo-controlled RCTs, INPULSIS 1 and INPULSIS 2 (16). A combined total of 1,066 patients were randomised to take either nintedanib or placebo over a 1-year period. Pooled analysis showed a significant reduction in annual decline in FVC (113.6 vs. $223.5 \mathrm{~mL}$ in placebo group). In similarity to pirfenidone, INPULSIS found that significantly fewer patients taking nintedanib had a FVC decline $>10 \%$. However, this study was not powered sufficiently to detect changes in mortality.

Nintedanib received US FDA approval on the same day as pirfenidone in October 2014 and EMA approval in November 2014. NICE approved use of nintedanib in the UK in January 2016 (17). As for pirfenidone, it is only licensed in the UK for patients with an FVC between $50-80 \%$ predicted. Nintedanib should also be stopped if there is a $>10 \%$ decline in FVC in a 1 -year period. These restrictions do not apply to the FDA or EMA licences. As with pirfenidone there is evidence in post-hoc studies that 
Table 1 Incidence of adverse events (\%) whilst taking pirfenidone in the CAPACITY (10) and ASCEND (11) trials

\begin{tabular}{lcc}
\hline Adverse event & CAPACITY & ASCEND \\
\hline Nausea & 36 & 36 \\
Rash & 32 & 28 \\
Dyspepsia & 19 & 17.6 \\
Dizziness & 18 & 17.6 \\
Vomiting & 14 & 12.9 \\
Anorexia & 11 & 15.8 \\
Insomnia & 16 & 11.2 \\
\hline
\end{tabular}

nintedanib is equally as effective in those with an FVC above $80 \%$ (18), although this treatment is not currently licensed by NICE in this patient group.

\section{Adverse effects of antifibrotic}

Both pirfenidone and nintedanib have similar effects on lung function with pirfenidone showing a mortality benefit in pooled data. The decision as to which treatment to start is based on joint decision making between patient and physician. It is important to discuss the intentions of treatment to slow decline or stabilise disease. Patient's expectations of a new treatment need to be addressed by advising them that the antifibrotic treatments have not been shown to improve breathlessness. One study has shown an improvement in cough with pirfenidone therapy (19). Strategies to manage symptoms with breathlessness management techniques, long term and ambulatory oxygen assessment and referral to pulmonary rehabilitation must occur in parallel to starting antifibrotic medication. The choice of antifibrotic is a joint decision between patient and physician after discussing the relative potential side effect profiles and contraindications of each treatment.

Pirfenidone is contra-indicated in renal failure (eGFR $<30 \mathrm{~mL} /$ minute $/ 1.73 \mathrm{~m}^{2}$ ). It is metabolised by cytochrome P450 1A2 (CYP1A2) and interacts with drugs which are metabolised by this pathway and as such should be avoided such as fluvoxamine, ciprofloxacin, propafenone and grapefruit. Pirfenidone may also interact with other CYP isoenzymes inhibitors (fluconazole, paroxetine, fluoxetine, amiodarone, chloramphenicol) and inducers (omeprazole, tobacco smoking). Patients are strongly advised to stop smoking due to CYP1A2 hepatic enzyme induction, which causes a significant increase in pirfenidone clearance.

Due to nintedanib's mechanism of action on platelet derived growth factor and the coagulation pathway there are concerns about its cardiovascular and bleeding risks (20). Nintedanib is therefore contra-indicated in patients with significant active cardiovascular disease and those taking dual anti-platelet therapy or warfarin. Post-trial and realworld data has not demonstrated any additional safety signals with respect to cardiovascular and bleeding risks $(16,21)$. Nintedanib is contraindicated in patients with peanut allergy. Both antifibrotics can cause elevation of liver enzymes and so liver function tests (LFTs) should be measured at baseline and throughout treatment.

Pirfenidone is manufactured as $267 \mathrm{mg}$ capsules and the dose is titrated from $267 \mathrm{mg}$ three times a day over 2 weeks to the maximal dose of $801 \mathrm{mg}$ three times a day, equating to three capsules three times a day. The advantage of this is that dose reductions and re-titrations can be employed to manage side effects. Nintedanib is dosed at a $150-\mathrm{mg}$ capsule twice a day with a $100-\mathrm{mg}$ dose for managing side effects. Some patients may find the pill burden with Pirfenidone challenging. However, a new formulation with an 801-mg capsule will help in alleviating these patient concerns.

Both pirfenidone and nintedanib have side effect profiles that require careful education and management to maintain adherence and maximise efficacy. Both the CAPACITY (10) and ASCEND (11) trials found similar side effect profiles with pirfenidone (Table 1). Adverse effects included nausea, vomiting, dyspepsia, anorexia and skin disorders such as rashes and photosensitivity. Most adverse events reported were classified as mild to moderate. Both trials showed that pirfenidone is generally well-tolerated with a favourable risk-benefit profile. Relative discontinuation rates due to adverse effects were $14.4 \%$ vs. $10.8 \%$ (pirfenidone $v$. placebo) in the ASCEND study (11) and $15 \%$ vs. $9 \%$ (pirfenidone vs. placebo) in the CAPACITY trials (10). The INPULSIS trials similarly showed that most adverse events experienced with nintedanib are mild to moderate in nature (16). Common adverse events include diarrhoea, nausea, vomiting and cough (Table 2). Reports of serious adverse events were similar between nintedanib and placebo. Nintedanib was discontinued in $25.2 \%$ in the treatment group versus $17.6 \%$ in the placebo group in INPULSIS-1 and $23.7 \%$ vs. $17.6 \%$ in INPULSIS-2 (16). Adverse events were the most common reason cited for treatment discontinuation. However, despite diarrhoea being the most commonly reported adverse event with nintedanib, less than $5 \%$ of patients with diarrhoea discontinued treatment in 
Table 2 Incidence of adverse events (\%) whilst taking nintedanib in the INPULSIS-1 and INPULSIS-2 trials (16)

\begin{tabular}{lcc}
\hline Adverse event & INPULSIS-1 & INPULSIS-2 \\
\hline Diarrhoea & 61.5 & 63.2 \\
Nausea & 22.7 & 26.1 \\
Cough & 15.2 & 11.6 \\
Reduced appetite & 8.4 & 12.8 \\
Vomiting & 12.9 & 10.3 \\
Weight loss & 8.1 & 11.2 \\
\hline
\end{tabular}

both INPULSIS-1 and INPULSIS-2. Data from real world studies into the relative safety and adverse event profiles of pirfenidone and nintedanib $(21,22)$ corroborate the findings of ASCEND, CAPACITY and INPULSIS and suggest overall favourable safety outcomes.

\section{Managing antifibrotic medication adverse effects}

The majority of antifibrotic side effects are gastro-intestinal in nature. Pharmacokinetic studies with pirfenidone have shown that taking medication with food can reduce the peak dose (23). Patients are therefore advised to take tablets during a meal for both antifibrotics i.e., not on an empty stomach and divide the dosage across the meal (24). Ongoing side effects may require supervised dose reduction and re-titration once the symptoms have subsided. Indigestion should be treated with a proton-pump inhibitor (24). There is a theoretical risk of a pharmacokinetic interaction between pirfenidone and omeprazole (25) so this should therefore be avoided if using pirfenidone but may be given with nintedanib. Diarrhoea, particularly with nintedanib may be managed with rehydration and anti-diarrhoeal medication for example loperamide (26), but if persistent, may require dose reduction. Anti-emetics may also be used to treat nausea (27).

Pirfenidone may cause a skin rash or photosensitivity. Preventative advice should be given, including avoiding direct sun exposure, wearing protective clothing and applying UVA and UVB sunscreen factor 50 (28). A mild to moderate photosensitive reaction requires dose reduction for 7 days with recommencement of treatment if resolving. More severe reactions require pirfenidone to be stopped for 15 days and then carefully re-titrated when resolved (24). In a minority of patients, patients may become sensitised and so pirfenidone cannot be recommenced as the rash reoccurs even at lower doses.

\section{Managing symptoms related to IPF}

IPF patients have a high burden of symptoms with a significant impact on quality of life $(29,30)$. This may be a result of physical symptoms including fatigue, cough and breathlessness or psychosocial factors such as depression, anxiety, relationship problems and financial difficulties (29). An IPF diagnosis also has a significant impact on patient's caregivers (29). Following a diagnosis of IPF best supportive care should be offered in parallel to antifibrotic medication to help patients and their families maintain their quality of life and learn how to live with the complexities of the disease. Supportive care is required throughout the trajectory of the disease and patients should be supported at each step of the process. Qualitative research into patient's perspectives of IPF identifies health care practitioners as a vital tool in supporting patients (29). The use of patientcentred communication styles (31) as well as provision of advice and information about the disease (22) can aid patient management. This should include advice about how to manage any symptoms with an emphasis on breathlessness and cough management.

\section{Breatblessness}

Chronic breathlessness is frequent in patients with IPF and can trigger frequent hospital admissions. There has been a move towards non-pharmacological management of breathlessness. Alleviating psychosocial distress associated with breathlessness can aid symptom control and improve quality of life (32). The use of patient education, anxiety management techniques, practical support for carers and physiotherapy have all been shown to improve breathlessness control (32). The use of simple handheld fans can improve patient self-efficacy and shorten periods of breathlessness (33).

\section{Cough}

Cough in IPF tends to be non-productive and dry and may be present in up to $80 \%$ of patients (34). The underlying mechanism of cough in IPF is complex and likely multifactorial (35). Cough in IPF is usually severe, not easily amenable to treatment (34) and cough frequency in IPF is higher than other respiratory conditions including chronic obstructive pulmonary disease (COPD) and asthma (36,37). Cough has a significant impact on a patient's quality of life 
and may lead to sleeping difficulties (38), decreased social interaction and relationship problems (39). Unfortunately, there is little evidence base for effective cough treatments in IPF. Conventional cough suppressant remedies often have no benefit (34). Gastro-oesophageal reflux disease (GORD) has been suggested as a potential contributor to the pathophysiology of IPF and cough (40). However, in one study the use of acid-suppressing medication did not improve cough and led to a paradoxical increase in non-acid reflux (41). Corticosteroids have been shown to improve cough in one small RCT and may be used at a tapering dose if beneficial (34). Opiates may be used in a palliative setting but there is no evidence for their effectiveness in IPF for cough suppression (42). The use of pirfenidone has been shown to have a possible beneficial effect on cough in a subgroup population of IPF patients (19). Cough can be a debilitating symptom in IPF with limited evidence based therapies and thus needs to be a major focus of research.

\section{Ambulatory oxygen therapy}

The use of ambulatory oxygen in IPF remains controversial. It is aimed at improving exercise capacity and relieving symptoms of breathlessness. There are a handful of small studies which suggest use of supplemental oxygen in IPF may improve both exercise capacity and breathlessness (43-45). The outcome of a RCT investigating the usefulness of oxygen in fibrotic lung disease will hopefully help generate guidelines on the use of ambulatory oxygen in IPF (46). However, ambulatory oxygen may have a negative impact on patient's quality of life by physically restricting daily activities and making their condition more visible (47). Patients sometimes view the introduction of oxygen as a negative landmark and so delay its implementation (48). Timely referral for ambulatory oxygen therapy assessment is recommended in the NICE quality standards for IPF in the UK (49). The need for oxygen can be determined by regular lung function evaluation, the presence of hypoxia, and the 6-minute walk test.

\section{Pulmonary rehabilitation}

All IPF patients should be referred and have access to pulmonary rehabilitation (49). This consists of a 6- or 8 -week programme which encompasses both exercise and education sessions. Pulmonary Rehabilitation improves clinical outcomes and symptoms in IPF, improving exercise tolerance and quality of life (50). One meta-analysis of
RCT's found an overall improvement in the 6-minute walking distance test of $27.4 \mathrm{~m}$ in those undertaking pulmonary rehabilitation (51). Provision of pulmonary rehabilitation for IPF patients can be patchy and is delivered largely via COPD targeted programmes. Improved access for IPF patients and condition specific programmes are key targets for improving IPF care.

\section{Managing the psychological impact of disease}

Appropriate psychological support is also important in IPF. Anxiety occurs in approximately two-thirds of patients with IPF (52). Depression is also a common co-morbidity. The progressive nature of IPF, with increasing shortness of breath and reduction in patient's ability to complete daily activities can significantly impair emotional well-being. Many patients wish to receive psychological support which may be accessed through the MDT or general practitioner. Cognitive-behavioural therapy has evidence for efficacy in COPD and this may be a useful technique in IPF (53). Pharmacological interventions for anxiety and depression also have a role, such as the use of selective serotonin reuptake inhibitors (SSRIs) (54).

\section{End of life care}

IPF patients require ongoing palliative care strategies commencing close to diagnosis. This should include introduction to community palliative and symptom management services, discussions around their priorities of care and support for their family and friends who may feel very helpless (55). In the UK, guidelines recommend that discussions around end of life should be started at the time of diagnosis (49). The majority of patients would like to know everything about their condition and its prospects, including prognostic information (52). Discussions may include likely disease trajectory, communication and coordination of care and end of life preferences. However, end of life discussions can be very distressing for both patient and partner's (56) and so the delivery of information should be personalised to individual patient's needs.

\section{The crucial role of the ILD nurse specialists}

ILD specialist nurses are pivotal to the overall care of patients with IPF. UK guidance suggests all patients and partners affected by IPF should have access to a specialist nurse through all phases of the disease (49). ILD specialist nurses 
are instrumental in their involvement throughout the patient journey from initial diagnosis, continued management and during the final stages of end of life care. The ILD specialist nurses act as an advocate for the patient and are heavily involved in the implementation of management plans. They provide patients with information about the disease, discuss care options and discuss medication/side effects and oxygen supply. In one study, $88 \%$ of respondents found ILD specialist nurses beneficial (52). Follow up care for IPF does vary across the UK (57). However dedicated support instituted in nurse led clinics, both face to face and via telephone consultation, can provide support on adherence and side effect management, and help to guide the care pathway initiated in ILD specialist centres. The introduction of support from ILD specialist nurses has been shown to increase concordance with treatment (58), reduces the need for dose reduction/interruption and reduces discontinuation of antifibrotic (24). The ILD specialist nurses provide an important link between the MDT and the general practitioner. Specialist nurses and support strategies such as IPF care (59) provide a key resource and support for patients. Patients not only welcome support regarding antifibrotic side effect management but also welcome discussions regarding oxygen, pulmonary rehabilitation and palliative care (59).

\section{Conclusions}

Over the past decade there have been significant changes to the management of IPF following the advent of two antifibrotic therapies. Pirfenidone and nintedanib offer potential prognostic benefit in an otherwise deadly disease. Both drugs have significant but manageable side effect profiles and specialists managing patients with IPF need to take this into consideration when selecting the right drug for the right patient. Symptom control measures need to continue alongside antifibrotic therapy and the ILD specialist nurse is key to ensuring a holistic approach is adopted.

\section{Acknowledgements}

None.

\section{Footnote}

Conflicts of Interest: N Chaudhuri has received educational funding and project grants from Boehringer Ingelheim, Intermune and Roche. These funds are not related to this publication. The other authors have no conflicts of interest to declare.

\section{References}

1. Center for Drug Evaluation and Research (CDER), U.S. Food and Drug Administration (FDA). The Voice of the Patient. A series of reports from the U.S. Food and Drug Administration's (FDA's) Patient-Focused Drug Development Initiative. Idiopathic Pulmonary Fibrosis. Available online: https://www.fda.gov/downloads/ ForIndustry/UserFees/PrescriptionDrugUserFee/ UCM440829.pdf

2. British Lung Foundation. The battle for breath - the impact of lung disease in the UK. Available online: https:// www.blf.org.uk/what-we-do/our-research/the-battle-forbreath-2016

3. Hutchinson J, Fogarty A, Hubbard R, et al. Global incidence and mortality of idiopathic pulmonary fibrosis: a systematic review. Eur Respir J 2015;46:795-806.

4. Navaratnam V, Fleming KM, West J, et al. The rising incidence of idiopathic pulmonary fibrosis in the UK. Thorax 2011;66:462-7.

5. Ley B, Collard HR, King TE. Clinical Course and Prediction of Survival in Idiopathic Pulmonary Fibrosis. Am J Respir Crit Care Med 2011;183:431-40.

6. Demedts M, Behr J, Buhl R, et al. High-dose acetylcysteine in idiopathic pulmonary fibrosis. $\mathrm{N} \mathrm{Engl} \mathrm{J}$ Med 2005;353:2229-42.

7. Idiopathic Pulmonary Fibrosis Clinical Research Network, Raghu G, Anstrom KJ, et al. Prednisone, azathioprine, and $\mathrm{N}$-acetylcysteine for pulmonary fibrosis. N Engl J Med 2012;366:1968-77.

8. du Bois RM, Weycker D, Albera C, et al. Ascertainment of individual risk of mortality for patients with idiopathic pulmonary fibrosis. Am J Respir Crit Care Med 2011;184:459-66.

9. Oku H, Shimizu T, Kawabata T, et al. Antifibrotic action of pirfenidone and prednisolone: different effects on pulmonary cytokines and growth factors in bleomycininduced murine pulmonary fibrosis. Eur J Pharmacol 2008;590:400-8.

10. Noble PW, Albera C, Bradford WZ, et al. Pirfenidone in patients with idiopathic pulmonary fibrosis (CAPACITY): two randomised trials. Lancet 2011;377:1760-9.

11. King Jr TE, Bradford WZ, Castro-Bernardini S, et al. (2014). A phase 3 trial of pirfenidone in patients with idiopathic pulmonary fibrosis. $\mathrm{N}$ Engl J Med 2014;370:2083-92.

12. Nathan SD, Albera C, Bradford WZ, et al. Effect of pirfenidone on mortality: pooled analyses and meta- 
analyses of clinical trials in idiopathic pulmonary fibrosis. Lancet Respir Med 2017;5:33-41.

13. National Institute for Health and Care Excellence. Pirfenidone for treating idiopathic pulmonary fibrosis (review of TA282). London: NICE, 2013.

14. Albera C, Costabel U, Fagan EA, et al. Efficacy of pirfenidone in patients with idiopathic pulmonary fibrosis with more preserved lung function. Eur Respir J 2016;48:843-51.

15. Wollin L, Wex E, Pautsch A, et al. (2015). Mode of action of nintedanib in the treatment of idiopathic pulmonary fibrosis. Eur Respir J 2015;45:1434-45.

16. Richeldi L, du Bois RM, Raghu G, et al. Efficacy and safety of nintedanib in idiopathic pulmonary fibrosis. $\mathrm{N}$ Engl J Med 2014;370:2071-82.

17. National Institute for Health and Care Excellence. Nintedanib for treating idiopathic pulmonary fibrosis (TA379). London: NICE, 2016.

18. Maher TM, Flaherty KR, Noble PW, et al. Effect of baseline FVC on lung function decline with nintedanib in patients with IPF. Eur Respir J 2015;46:OA4499.

19. Azuma A, Taguchi $Y$, Ogura T, et al. Exploratory analysis of a phase III trial of pirfenidone identifies a subpopulation of patients with idiopathic pulmonary fibrosis as benefiting from treatment. Respir Res2011;12:143.

20. Kamba T, McDonald DM. Mechanisms of adverse effects of anti-VEGF therapy for cancer. Br J Cancer 2007;96:1788-95.

21. Hughes G, Toellner H, Morris H, et al. Real World Experiences: Pirfenidone and Nintedanib are Effective and Well Tolerated Treatments for Idiopathic Pulmonary Fibrosis. J Clin Med 2016;5:78.

22. Galli JA, Pandya A, Vega-Olivo M, et al. Pirfenidone and nintedanib for pulmonary fibrosis in clinical practice: Tolerability and adverse drug reactions. Respirology 2017;22:1171-8.

23. Rubino CM, Bhavnani SM, Ambrose PG, et al. Effect of food and antacids on the pharmacokinetics of pirfenidone in older healthy adults. Pulm Pharmacol Ther 2009;22:279-85.

24. Costabel U, Bendstrup E, Cottin V, et al. Pirfenidone in idiopathic pulmonary fibrosis: expert panel discussion on the management of drug-related adverse events. Adv Ther 2014;31:375-91.

25. Cottin V, Crestani B, Valeyre D, et al. Diagnosis and management of idiopathic pulmonary fibrosis: French practical guidelines. Eur Respir Rev 2014;23:193-214.

26. Mazzei ME, Richeldi L, Collard HR. Nintedanib in the treatment of idiopathic pulmonary fibrosis. Ther Adv Respir Dis 2015;9;121-9.

27. Edwards IR, Aronson JK. Adverse drug reactions: definitions, diagnosis, and management. Lancet 2000;356:1255-9.

28. Drucker AM, Rosen CF. Drug-induced photosensitivity. Drug Saf 2011;34:821-37.

29. Russell AM, Ripamonti E, Vancheri C. Qualitative European survey of patients with idiopathic pulmonary fibrosis: patients' perspectives of the disease and treatment. BMC Pulm Med 2016;16:10.

30. Kotecha J, Atkins C, Wilson A. Patient confidence and quality of life in idiopathic pulmonary fibrosis and sarcoidosis. Sarcoidosis Vasc Diffuse Lung Dis 2016;33:341-8.

31. Wuyts WA, Peccatori FA, Russell AM. Patient-centred management in idiopathic pulmonary fibrosis: similar themes in three communication models. Eur Respir Rev 2014;23:231-8.

32. Booth S, Moffat C, Farquhar M, et al. Developing a breathlessness intervention service for patients with palliative and supportive care needs, irrespective of diagnosis. J Palliat Care 2011;27:28.

33. Galbraith S, Fagan P, Perkins P, et al. Does the use of a handheld fan improve chronic dyspnea? A randomized, controlled, crossover trial. J Pain Symptom Manage 2010;39:831-8.

34. Hope-Gill BD, Hilldrup S, Davies C, et al, NK. A study of the cough reflex in idiopathic pulmonary fibrosis. Am J Respir Crit Care Med 2003;168:995-1002.

35. van Manen MJ, Birring SS, Vancheri C, et al. Cough in idiopathic pulmonary fibrosis. Eur Respir Rev 2016;25:278-86.

36. Key AL, Holt K, Hamilton A, et al. Objective cough frequency in idiopathic pulmonary fibrosis. Cough 2010;6:4 .

37. Sumner H, Woodcock A, Kolsum U, et al. Predictors of objective cough frequency in chronic obstructive pulmonary disease. Am J Respir Crit Care Med 2013;187:943-9.

38. Swigris JJ, Stewart AL, Gould MK, et al. Patients' perspectives on how idiopathic pulmonary fibrosis affects the quality of their lives. Health Qual Life Outcomes 2005;3:61.

39. Morice AH, Fontana GA, Belvisi MG, et al. ERS guidelines on the assessment of cough. Eur Respir J 2007:29:1256-76.

40. Perng DW, Chang KT, Su KC, et al. Exposure of airway 
epithelium to bile acids associated with gastroesophageal reflux symptoms: a relation to transforming growth factor- $\beta 1$ production and fibroblast proliferation. Chest 2007;132:1548-56.

41. Kilduff CE, Counter MJ, Thomas GA, et al. Effect of acid suppression therapy on gastroesophageal reflux and cough in idiopathic pulmonary fibrosis: an intervention study. Cough 2014;10:4.

42. Harrison, NK. Cough, sarcoidosis and idiopathic pulmonary fibrosis: raw nerves and bad vibrations. Cough 2013;9:9.

43. Visca D, Montgomery A, de Lauretis A, et al. Ambulatory oxygen in interstitial lung disease. Eur Respir J 2011;38:987-90.

44. Frank RC, Hicks S, Duck AM, et al. Ambulatory oxygen in idiopathic pulmonary fibrosis: of what benefit? Eur Respir J 2012;40:269-70.

45. Sharp C, Adamali H, Millar AB. Ambulatory and shortburst oxygen for interstitial lung disease. Cochrane Database Syst Rev 2016;7:CD011716.

46. Visca D, Tsipouri V, Mori L, et al. Ambulatory oxygen in fibrotic lung disease (AmbOx): study protocol for a randomised controlled trial. Trials 2017;18:201.

47. Wuyts WA, Peccator FA, Russell AM. Patient-centred management in idiopathic pulmonary fibrosis: similar themes in three communication models. Eur Respir Rev 2014;23:231-8.

48. van Manen MJ, Geelhoed JM, Tak NC, et al. Optimizing quality of life in patients with idiopathic pulmonary fibrosis. Ther Adv Respir Dis 2017;11:157-69.

49. National Institute for Health and Care Excellence. Idiopathic pulmonary fibrosis in adults: diagnosis and management (CG163). London: NICE, 2013.

50. Swigris JJ, Brown KK, Make BJ, et al. Pulmonary rehabilitation in idiopathic pulmonary fibrosis: a call for continued investigation. Respir Med 2008;102:1675-80.

51. Bajwah S, Ross JR, Peacock JL, et al. Interventions to

Cite this article as: Shaw J, Marshall T, Morris H, Hayton C, Chaudhuri N. Idiopathic pulmonary fibrosis: a holistic approach to disease management in the anti-fibrotic age. J Thorac Dis 2017;9(11):4700-4707. doi: 10.21037/jtd.2017.10.111 improve symptoms and quality of life of patients with fibrotic interstitial lung disease: a systematic review of the literature. Thorax 2013;68:867-79.

52. Van Manen M, Kreuter M, Van Den Blink B. What patients with pulmonary fibrosis and their partners really think; live patient and partner survey on pulmonary fibrosis in the Netherlands and Germany. Paper presented at A41 Here There and Everywhere: ILD Observational Studies. New York, NY: American Thoracic Society, 2015.

53. Heslop K, De Soyza A, Baker CR, et al. Using individualised cognitive behavioural therapy as a treatment for people with COPD. Nurs Times 2009;105:14-7.

54. National Institute for Health and Care Excellence.

Generalized anxiety disorder and panic disorder (with or without agoraphobia) in adults: management in primary, secondary and community care (CG113). London: NICE, 2011.

55. Bajwah S, Higginson IJ, Ross JR, et al. The palliative care needs for fibrotic interstitial lung disease: a qualitative study of patients, informal caregivers and health professionals. Palliat Med 2013;27:869-76.

56. Bajwah S, Koffman J, Higginson IJ, et al. 'I wish I knew more ...' the end-of-life planning and information needs for end-stage fibrotic interstitial lung disease: views of patients, carers and health professionals. BMJ Support Palliat Care 2013;3:84-90.

57. British Lung Foundation. IPF: the patient experience in England. Available online: https://www.blf.org.uk/ sites/default/files/BLF-IPF-Report-2015---Lost-in-theSystem---250215.pdf

58. Chaudhuri N, Duck A, Frank R, et al. "Real world experiences: pirfenidone is well tolerated in patients with idiopathic pulmonary fibrosis." Respir Med 2014;108:224-6.

59. Duck A, Spencer LG, Bailey S, et al. Perceptions, experiences and needs of patients with idiopathic pulmonary fibrosis. J Adv Nurs 2015;71:1055-65. 